\title{
Potential mechanism of ferroptosis in pancreatic cancer (Review)
}

\author{
GANG CHEN $^{1}$, GUANGQI GUO ${ }^{1}$, XIAODONG ZHOU $^{1}$ and HONGXIA CHEN ${ }^{2}$ \\ Departments of ${ }^{1}$ Gastroenterology, and ${ }^{2}$ Obstetrics and Gynecology, The First Affiliated Hospital of Nanchang University, \\ Nanchang, Jiangxi 330006, P.R. China
}

Received February 23, 2019; Accepted July 26, 2019

DOI: $10.3892 / 01.2019 .11159$

\begin{abstract}
Despite the incidence rates of pancreatic cancer being low worldwide, the mortality rates remain high. To date, there is no effective drug treatment for pancreatic cancer. Numerous signalling pathways and cytokines regulate the occurrence and development of pancreatic cancer. Ferroptosis is a non-traditional form of cell death resulting from iron-dependent lipid peroxide accumulation. Studies have demonstrated that ferroptosis is associated with a variety of different types of cancer, such as breast cancer, hepatocellular carcinoma and pancreatic cancer. The present study demonstrated that ferroptosis controls the growth and proliferation of pancreatic cancer, providing a new approach for the treatment of pancreatic cancer. Iron metabolism and reactive oxygen species metabolism are the key pathways involved in ferroptosis in pancreatic cancer. In addition, a number of regulators of ferroptosis, such as glutathione peroxidase 4 and the cystine/glutamate antiporter system Xc-, also play pivotal roles in the regulation of ferroptosis. In the present review, the regulatory mechanisms associated with ferroptosis in pancreatic
\end{abstract}

Correspondence to: Professor Xiaodong Zhou, Department of Gastroenterology, The First Affiliated Hospital of Nanchang University, 17 Yongwaizheng Street, Nanchang, Jiangxi 330006, P.R. China

E-mail: yfyzxd@163.com

Professsor Hongxia Chen, Department of Obstetrics and Gynecology, The First Affiliated Hospital of Nanchang University, 17 Yongwaizheng Street, Nanchang, Jiangxi 330006, P.R. China E-mail: yfychx@163.com

Abbreviations: ATF4, transcription factor 4; ART, artesunate; BECN1, Beclin 1; CN-A, Cotylenin A; $\mathrm{Fe}^{3+}$, ferric iron; $\mathrm{Fe}^{2+}$, ferrous iron; FtH, ferritin heavy chain; FtL, ferritin light chain; GSH, glutathione; GPX4, glutathione peroxidase 4; HSPA5, heat shock $70 \mathrm{kDa}$ protein 5 ; HSPB1, heat shock protein $\beta-1$; LOX, lipoxygenases; MDA, malondialdehyde production, NOX, NADPH oxidase; NRF2, nuclear factor (erythroid derived)-like 2; PDAC, pancreatic ductal adenocarcinoma; PEITC, phenethyl isothiocyanate; ROS, reactive oxygen species; RCD, regulated cell death; VDAC, mitochondrial voltage-dependent anion channel

Key words: ferroptosis, pancreatic cancer, iron, reactive oxygen species, treatment cancer are summarized, alongside other associated forms of digestive system cancer. The treatment of ferroptosis-based diseases is also addressed.

\section{Contents}

1. Introduction

2. Molecular mechanism underlying ferroptosis and relative regulators in cancer

3. Potential roles of ferroptosis in pancreatic cancer

4. Summary and perspective

\section{Introduction}

Pancreatic cancer is characterized by high mortality and metastasis rates (1); it is one of the four most common causes of cancer-associated mortality with a reported $6.6 \%$ in Europe in 2018 (2). Based on the GLOBOCAN 2018 data, the number of pancreatic cancer-associated deaths was 432,242 per year in the USA (3). Pancreatic ductal adenocarcinoma (PDAC) is the primary pathological type of pancreatic cancer, and patients with the disease present with a poor prognosis (4). A genetic study revealed that the Kirsten rat sarcoma viral oncogene (KRAS) mutation is an early event in pancreatic tumourigenesis and plays a significant role in pancreatic cancer (5). Currently, surgery is the only means of treating pancreatic cancer; however, it is not sufficient to improve the survival rate of patients with pancreatic cancer $(6,7)$. Unfortunately, traditional chemotherapy drugs are not effective due to the resistance of pancreatic cancer cells. Despite the fact that the prognosis has been enhanced by clinical standard therapies (8), the 5 year survival rates of patients with pancreatic cancer remain at $<5 \%$ (9). Thus, finding new therapeutic methods for pancreatic cancer is imperative for future preclinical research.

Ferroptosis, a recently discovered form of regulated cell death (RCD), is dependent on the presence of intracellular iron and the accumulation of reactive oxygen species (ROS) (10). Ferroptosis has been identified in numerous pathological diseases, such as ischaemia-reperfusion injury, neurodegenerative diseases and a series of different cancer types, including breast cancer, hepatocellular carcinoma and pancreatic cancer (10-12). Recently, a number of clinical studies have indicated that traditional Chinese herbs exhibit potent anticancer effects in pancreatic cancer by affecting 
ferroptosis, which suggests that ferroptosis may also play an important role in the disease (13-15). Furthermore, it has been demonstrated that ferritinophagy, the lipid peroxidation pathway, the glutathione (GSH) peroxidase 4 (GPX4) pathway and the system Xc- pathway are closely associated with ferroptosis.

In the present review article, the potential molecular mechanisms underlying ferroptosis in pancreatic cancer are discussed, alongside the potential future directions for ferroptosis research.

\section{Molecular mechanism underlying ferroptosis and relative regulators in cancer}

In 2012, a new form of RCD called ferroptosis was discovered and reported by Dixon et al (10). Ferroptosis is morphologically and mechanistically different from apoptosis, necroptosis, autophagy and other forms of cell death. Morphologically, it has been demonstrated that in ferroptosis, the mitochondrial volume is decreased compared with that of normal mitochondria, the mitochondrial membrane density is increased, the mitochondrial ridge disappears and the outer membrane ruptures (16). Biochemically, ferroptosis is dependent on iron and ROS, which are characteristic of lipid peroxidation (17). Currently, studies indicate that the two main pathways involved in ferroptosis are the iron metabolism pathway and the ROS metabolism pathway $(16,18)$. Iron metabolism in the cell consists of the import, storage and export of iron. Ferric iron $\left(\mathrm{Fe}^{3+}\right)$ bound to transferrin is transported to the endosome via transferrin receptor 1 . Inside the endosome, $\mathrm{Fe}^{3+}$ is reduced to ferrous iron $\left(\mathrm{Fe}^{2+}\right)$, which is finally gathered in a labile iron pool in the cytoplasm. Cytoplasmic iron is retained as ferritin, which comprises ferritin heavy chain $(\mathrm{FtH})$ and ferritin light chain (FtL). Finally, excessive iron is exported by ferroportin $(19,20)$. Ferroptosis is mediated by the Fenton reaction, in which $\mathrm{Fe}^{2+}$ reacts with hydrogen peroxide to generate ROS (21). ROS are a very important secondary signal in cells, and are formed by the partial reduction of molecular oxygen, including superoxide $\left(\mathrm{O}_{2}{ }^{-}\right)$, peroxides $\left(\mathrm{H}_{2} \mathrm{O}_{2}\right.$ and $\mathrm{ROOH}$ ) and free radicals ( $\mathrm{HO}^{\circ}$ and $\left.\mathrm{RO}^{\circ}\right)$ (17). ROS damage the stability of DNA and promote cell death. ROS-induced ferroptosis may involve multiple sources. In addition to the iron-dependent accumulation of ROS, NADPH-dependent lipid peroxidation and GSH depletion are known for the induction of ferroptosis $(10,22)$. Mechanistically, several molecules, called ferroptosis regulators, have recently been identified to regulate ferroptosis by targeting iron metabolism and lipid peroxidation. Among them, system Xc- and GPX4 are negative regulators of ferroptosis $(22,23)$. The system Xc- is an anionic amino acid transport system composed of the twelve-pass transmembrane transport protein cystine/glutamate transporter (SLC7A11) and the single-pass transmembrane regulator protein $4 \mathrm{~F} 2$ cell-surface antigen heavy chain (SLC3A2). System Xc- imports extracellular cysteine to exchange intracellular glutamate. Therefore, the selective inhibition of system Xc- causes a decrease in intracellular cysteine. Decreasing GSH synthesis results in excessive toxic lipid ROS accumulation, which triggers ferroptosis at the molecular level (23). GPX4 can directly decrease phospholipid hydroperoxide and prevent lipid peroxidation-dependent cell death, which is an essential negative regulator of ferroptosis. GPX4 is necessary to remove fatty oxygen radical enzymes that can decrease the toxic lipid hydroperoxides (L-OOH) to lipid alcohols (L-OH). Once GPG4 is inactivated, L-OOH will gradually accumulate. At the same time, cellular $\mathrm{L}-\mathrm{OOH}$ is catalysed by iron into toxic lipid radicals, such as the alkoxy radical L-O, resulting in cytotoxicity and cell death (22). By contrast, voltage-dependent anion channel (VDAC)2/3 and NADPH oxidase (NOX), as positive regulators, promote ferroptosis. Mitochondrial voltage-dependent anion channels (VDACs) are novel targets for anticancer drugs. Cells with more VDAC protein are more sensitive to erastin (24). Erastin, the classical inducer of ferroptosis, interacts with VDAC proteins, leading to mitochondrial dysfunction, the release of oxidative species and non-apoptotic oxidative cell death (25). The NOX protein family reduces oxygen to superoxide by transferring electrons across biological membranes. The canonical NOX inhibitor diphenyleneiodonium and the NOX1/4-specific inhibitor GKT137831 were both shown to suppress erastin-induced ferroptosis in Calu-1 cells in a preivious study (10). The protein cellular tumour antigen p53 (p53) participates in controlling cell survival and death, and plays double roles in regulating ferroptosis through a transcriptional or post-translational mechanism. Spermidine/spermine N1-acetyltransferase 1 (SAT1) is an important regulator of polyamine metabolism through acetylating spermidine and spermine using acetyl-coenzyme A (26). The expression of glutaminase 2 (GLS2) is responsible for p53-mediated oxygen consumption, mitochondrial respiration and ATP generation in cancer cells (27). p53 promotes ferroptosis by inhibiting SLC7A11 expression and increasing SAT1 and GLS2 expression, while p53 also inhibits ferroptosis by downregulating the expression of DPP4 and upregulating the expression of CDKN1A/p21 (28). In other words, ferroptosis is a non-apoptotic form of cell death and is characterized by iron-dependent and ROS-dependent processes.

Ferroptosis is associated with a variety of physiological and pathological processes, including neurodegenerative disease, acute kidney failure, drug-induced hepatotoxicity, hepatic and heart ischaemia/reperfusion injury, and T-cell immunity, particularly in cancer cell death $(16,22,29-33)$. Ferroptosis has been identified in a number of different types of tumour cell, including breast cancer (34), head and neck cancer (35), hepatocellular carcinoma (36), pancreatic cancer (15) and ovarian cancer (37). Therefore, preventing ferroptosis has become an important strategy to prevent associated diseases and cancer types.

\section{Potential roles of ferroptosis in pancreatic cancer}

In recent years, it has proven difficult to identify new ways to treat pancreatic cancer. Gemcitabine, as the first-line drug, is used alone or in combination for the treatment of patients with advanced PDAC. Heat shock 70-kDa protein 5 (HSPA5) improves the anticancer activity of gemcitabine by inducing ferroptosis (38). In addition to gemcitabine, it has been demonstrated that certain traditional Chinese herbs induce ferroptosis in pancreatic cancer. Furthermore, a number of molecules have been demonstrated to induce ferroptosis in pancreatic cancer cells, suggesting that they may offer new options for pancreatic cancer treatment. 
Iron metabolism and ferroptosis in pancreatic cancer Ferritinophagy can promote ferroptosis in pancreatic cancer. A number of studies have provided evidence to support the association between autophagy and ferroptosis $(39,40)$. However, the underlying molecular mechanism between autophagy and ferroptosis in pancreatic cancer remains unclear. Recently, researchers have revealed that nuclear receptor coactivator 4 (NCOA4)-mediated ferritinophagy is an autophagic process that contributes to ferroptosis via the degradation of ferritin in pancreatic cancer $(41,42)$. The iron storage protein ferritin consists of two subunits of ferritin, FtH and FtL, which are associated with intracellular iron storage and release (43). NCOA4 is a cargo receptor specifically responsible for the selective autophagic turnover of ferritin in ferritinophagy. Degradation of ferritin leads to increased intracellular free iron, which triggers ROS generation and consequent ferroptosis in pancreatic cancer. Notably, knockdown of NCOA4 by specific shRNAs in pancreatic cancer inhibited ferritinophagy and suppressed erastin-induced ferroptosis. By contrast, overexpression of NCOA4 increased ferritin degradation and promoted ferroptosis. Knockout or knockdown of autophagy-related 5 (Atg5) and Atg7 in human pancreatic cancer cell lines decreased both intracellular $\mathrm{Fe}^{2+}$ and the product of $\mathrm{L}-\mathrm{OOH}$, and induced ferroptosis, which indicates that the Atg genes play an essential role in the mediation of autophagy and ferroptosis (42). Consequently, autophagy plays a key role in promoting ferroptosis, and further research is required in order to elucidate the association of ferritinophagy and ferroptosis, which can provide innovative treatments for pancreatic cancer.

Artesunate can induce ferroptosis in PDAC. The natural compound Artesunate (ART) is a noteworthy anti-malaria drug. It was previously demonstrated that ART also exhibited an anti-tumour effect and was a specific inducer of ferroptosis in a number of different types of cancer, including pancreatic cancer $(14,44,45)$. ART exhibited higher cytotoxicity in PDAC cells with Ras mutation compared with that in PDAC cell lines expressing wild-type KRas (14). Despite the fact that the underlying molecular mechanism is currently unknown, it was discovered that the functional lysosome and iron metabolism are involved in the ferroptosis induced by ART in PDAC and other types of tumour cell $(14,45)$. Ferritin binds with NCOA4 in the autophagosome and is delivered into the lysozyme, and ART accumulates in the lysosomes and increases ferritin protein degradation (44). Degradation of ferritin in lysosomes increases the volume of intracellular iron and plays an essential role in ART-activated ferroptosis, which is similar to ferritinophagy $(44,46)$. Yang et al (44) discovered that ART activates lysosomal activity by increasing V-ATPase assembly. Notably, the co-treatment of ART and transferrin increased lysosomal free iron and promoted ferroptosis in PDAC (14). Iron-dependent ROS generation and accumulation are indispensable steps for ferroptosis in response to ART $(44,46)$. In summary, ferroptosis induced by ART is dependent on the presence of intracellular iron.

\section{ROS metabolism and ferroptosis in pancreatic cancer}

LOX is sufficient for ferroptosis in pancreatic cancer. Recently, a very important association between lipid peroxide and ferroptosis has been identified. Decreased levels of GSH results in a deficiency of GPX4-reducing substrates, preventing the conversion of $\mathrm{L}-\mathrm{OOH}$ into $\mathrm{L}-\mathrm{OH}$. The gradual accumulation of L-OOH leads to ferroptosis (23). Two principal mechanisms for the formation of L-OOH are well known: Free radical chain oxidation of organic compounds, called autoxidation, and iron-dependent lipoxygenase (LOX)-mediated activity $(47,48)$. A recent study by Xie et al (13) demonstrated that the $12 / 15$-LOX inhibitor baicalein is effective in preventing erastin-induced ferroptosis by protecting pancreatic cancer cells from RSL3 toxicity, which indicates that LOX may regulate ferroptosis. Another study also reported that LOX activity may contribute to ferroptosis (49). LOXs are non-haem iron-containing dioxygenases that catalyse polyunsaturated fatty acids to produce fatty acid hydroperoxides that damage cells (37). Shintoku et al (50) demonstrated that upon exposure to the ferroptosis inducers erastin and RSL3, $\omega$-6 PUFA-mediated production of 4-hydroxy-2-nonenal was increased, contributing to ferroptosis, whereas inhibition or silencing of arachidonate 15-lipoxygenase (ALOX15) decreased both erastin-induced and RSL3-induced ferroptotic cell death in pancreatic cancer. During the process of inducing ferroptosis, the ALOX15 protein consistently localized to cellular membranes, suggesting that ALOX15 results in ferroptosis by inducing the production of $\mathrm{L}-\mathrm{OOH}$ in cell membranes (50). LOX is expressed in different kinds of tissue and is associated with several different types of cancer. Therefore, the induction of ferroptosis may be a new approach in treating cancer by regulating the expression of LOX (51). In conclusion, these reports implicated LOX as a key regulator of ferroptosis in PDAC.

Preventing mitochondrial lipid oxidation suppresses ferroptosis in pancreatic cancer. It is well known that the occurrence of ferroptosis is accompanied by morphological changes to the mitochondria (10). Previous studies have supported a hypothesis that ferroptosis is intrinsically associated with a lipid oxidation pathway by intersecting with the mitochondrial membrane $(52,53)$. Recently, Krainz et al (54) revealed that, as lipid peroxidation mitigators, XJB-5-131, JP4-039 and selected analogues influence the relative subcellular localization of nitroxide and prevent ferroptosis in PANC-1 cells. Both XJB-5-131 and JP4-039, mitochondrially targeted nitroxides, could prevent ROS accumulation and protect against mitochondrial function. The protective effect of JP4-039 was $>20$ - to 30-fold weaker than that of XJB-5-131, which coincides with the much lower concentration of JP4-039 in mitochondrial enrichment than that of XJB-5-131. These results suggest that mitochondria-targeted nitroxides inhibit ferroptosis, and that preventing mitochondrial lipid oxidation may offer a potential therapeutic opportunity in pancreatic cancer (54). To conclude, preventing mitochondrial lipid oxidation protects against ferroptosis in pancreatic cancer.

Non-oxidative dopamine inhibits ferroptosis by modulating lipid peroxidation in pancreatic cancer. It is already known that lipid peroxidation is an important element in inducing ferroptosis (17). Erastin is a classic ferroptosis inducer that inhibits system Xc-activity and VDAC (16). Dopamine is a powerful antioxidant and has numerous functions in the nervous and immune systems $(55,56)$. However, the effect 
of dopamine on ferroptosis is currently unknown. A recent study revealed that non-oxidative dopamine is an inhibitor of ferroptosis that protects against erastin-induced ferroptosis in pancreatic cancer (53). The levels of $\mathrm{Fe}^{2+}$ and malondialdehyde production (MDA), one of the end products of lipid peroxidation, were decreased following the dopamine treatment of erastin-induced cell death in pancreatic cancer. In terms of the mechanism, dopamine inhibits ferroptosis by modulating lipid peroxidation in two separate ways in PANC-1 cells. On the one hand, dopamine decreases the accumulation of $\mathrm{Fe}^{2+}$; on the other hand, dopamine markedly inhibits GSH depletion and GPX4 degradation (57). GPX4 is a GSH-dependent enzyme that cannot use GSH as a co-substrate to reduce lipid peroxidation due to GSH depletion. Both the iron-induced Fenton reaction and GSH depletion induce ferroptosis (22). In conclusion, these findings provide a new strategy for pancreatic cancer therapy by inhibiting lipid peroxidation.

Cotylenin A and phenethyl isothiocyanate induce ferroptosis by ROS accumulation in pancreatic cancer. Previous studies have demonstrated that certain drugs can induce ferroptosis in pancreatic cancer, providing a feasible therapeutic strategy against pancreatic cancer $(15,58)$. Phenethyl isothiocyanate (PEITC) is a potent generator of ROS, while cotylenin A (CN-A) exhibits potent anti-tumour activity in several cancer cell lines (59-63). A recent study by Kasukabe et al (15) demonstrated that, upon exposure to CN-A and PEITC, the proliferation of both PANC-1 cells and gemcitabine-resistant PANC-1 cells (PANC-1/GR) was inhibited by increasing ROS levels. Antioxidants (N-acetylcysteine and trolox), ferroptosis inhibitors (ferrostatin-1) and the iron chelator deferoxamine reverse this process, causing CN-A- and PEITC-induced ferroptosis in pancreatic cancer (15). Furthermore, it was also observed that CN-A and PEITC synergistically trigger more ROS accumulation when combined, compared with when used separately. These results suggest that CN-A and PEITC synergistically account for more ROS-ferroptosis pathway-mediated pancreatic cancer cell death compared with treatment with PEITC or CN-A alone. However, the molecular mechanisms underlying the interaction between CN-A and PEITC have not yet been elucidated in detail (15). Another study regarding the treatment of pancreatic cancer demonstrated similar evidence (58). Yamaguchi et al (58) reported that CN-A or sulfasalazine (SSZ) enhanced ROS production, which induced ferroptotic cell death in human pancreatic cancer. Above all, these findings provide a new drug treatment option for pancreatic cancer, and demonstrate that ROS are a vital hallmark of ferroptosis in pancreatic cancer.

\section{GPX4 and ferroptosis in pancreatic cancer}

GPX4 degradation promotes ferroptosis in pancreatic cancer. Recently a study revealed that baicalein negatively regulates ferroptosis by inhibiting the degradation of GPX4 (13). Baicalein, as a class of molecules present in certain traditional Chinese medical herbs, exhibits potent anticancer activities (64). In the study by Xie et al (13), it was demonstrated that, when compared with other well-known ferroptosis inhibitors (e.g., ferrostatin-1, liproxstatin-1, deferoxamine mesylate and $\beta$-mercaptoethanol), baicalein exhibited a greater level of anticancer activity in erastin-induced pancreatic cancer cell ferroptosis. Baicalein could inhibit ferroptosis by suppressing the degradation of GPX4 and decreasing the accumulation of iron (13). Furthermore, it was also revealed that, upon exposure to baicalein, the process of degrading erastin-induced nuclear factor (erythroid-derived 2)-like 2 (NRF2), a transcription factor that positively regulates the critical proteins of ferroptosis, such as GPX4, was inhibited (65). Qin et al (66) also demonstrated that baicalein modulates the NRF2/Keap1 system in both Keap1-independent and -dependent pathways to inhibit oxidative injury. Therefore, it can be concluded that baicalein may selectively activate the NRF2 pathway in pancreatic cancer. However, additional studies are required in order to clarify the precise molecular mechanisms underlying this process.

HSPA5-GPX4 pathway regulates ferroptosis in pancreatic cancer. According to their molecular mass, heat shock proteins (HSPs) are grouped into six families, namely, HSP100, HSP90, HSP70, HSP60, HSP40 and small HSPs. It is already known that HSP $\beta-1$ is a negative regulator of ferroptosis. Inhibition of heat shock factor 1-dependent HSPB1 expression and HSPB1 phosphorylation increased erastin-induced ferroptosis in human xenograft mouse tumour models $(67,68)$. Recently, Zhu et al (38) revealed that heat shock $70 \mathrm{kDa}$ protein 5 (HSPA5) is a negative regulator of ferroptosis in pancreatic cancer cells. HSPA5 is regulated by activating transcription factor 4 (ATF4), and both suppression and knockdown of ATF4 inhibit erastin-induced HSPA5 protein expression in PANC-1 cells and CFPAC1 cells. Upon activation of ATF4, HSPA5 protein binding to GPX4 increases the stability of GPX4 to protect against ferroptosis in pancreatic cancer. Following knockdown of HSPA5 or ATF4, MDA production was increased in erastin-induced cell death, demonstrating that ATF4-dependent HSPA5 expression inhibits ferroptosis through lipid peroxidation. Knockdown of HSPA5 and GPX4 in pancreatic cancer cells promotes ferroptosis in response to gemcitabine or pharmacological inhibition of the HSPA5-GPX4 pathway by epigallocatechin gallate or SSZ in pancreatic cancer cells rendered tumours more sensitive to gemcitabine both in vitro and in vivo. Furthermore, ferroptosis inhibitors reversed gemcitabine-induced cell death, which demonstrates that the HSPA5-GPX4 pathway regulates ferroptosis in pancreatic cancer cells (38). Above all, activation of the ATF4-HSPA5-GPX4 pathway protects from ferroptosis in pancreatic cancer cells and suggests a promising therapeutic strategy for pancreatic cancer.

\section{System Xc- and ferroptosis in pancreatic cancer}

Inhibiting system Xc-induces ferroptosis in pancreatic cancer. Sorafenib, a multikinase inhibitor, is currently recognized as the only anticancer drug in hepatocellular carcinoma treatment. In the process of treating hepatocellular carcinoma, ferroptosis plays a significant role in inducing hepatocellular carcinoma cell death (69). However, it is not yet certain whether sorafenib induces ferroptosis in human cancer cells originating from various tumours. Lachaier et al (70) demonstrated that sorafenib and erastin induced ferroptosis not only in hepatocellular carcinoma, but also in other types of cancer originating from tissues other than those of the pancreatic cancer. However, this process did not involve 

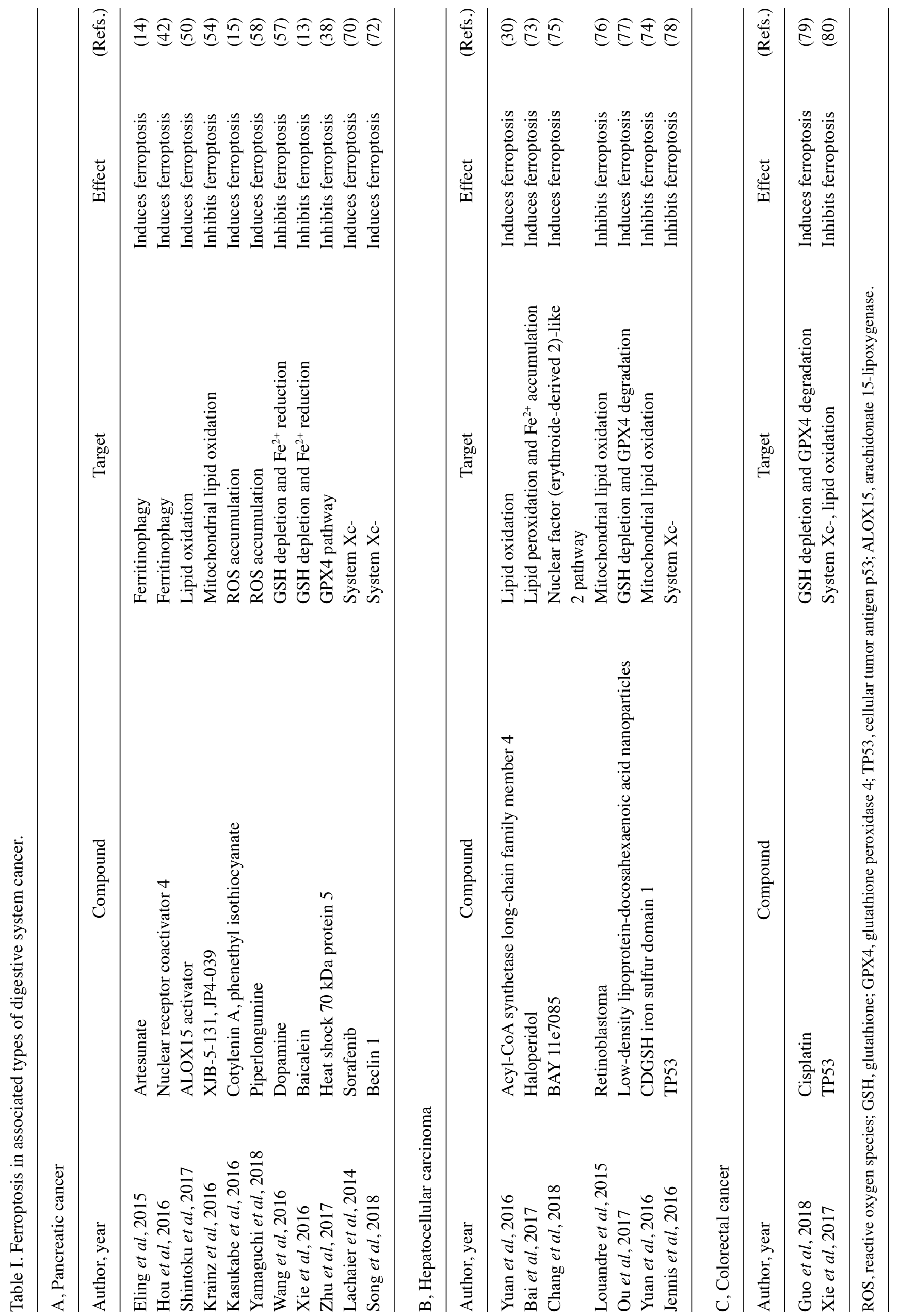


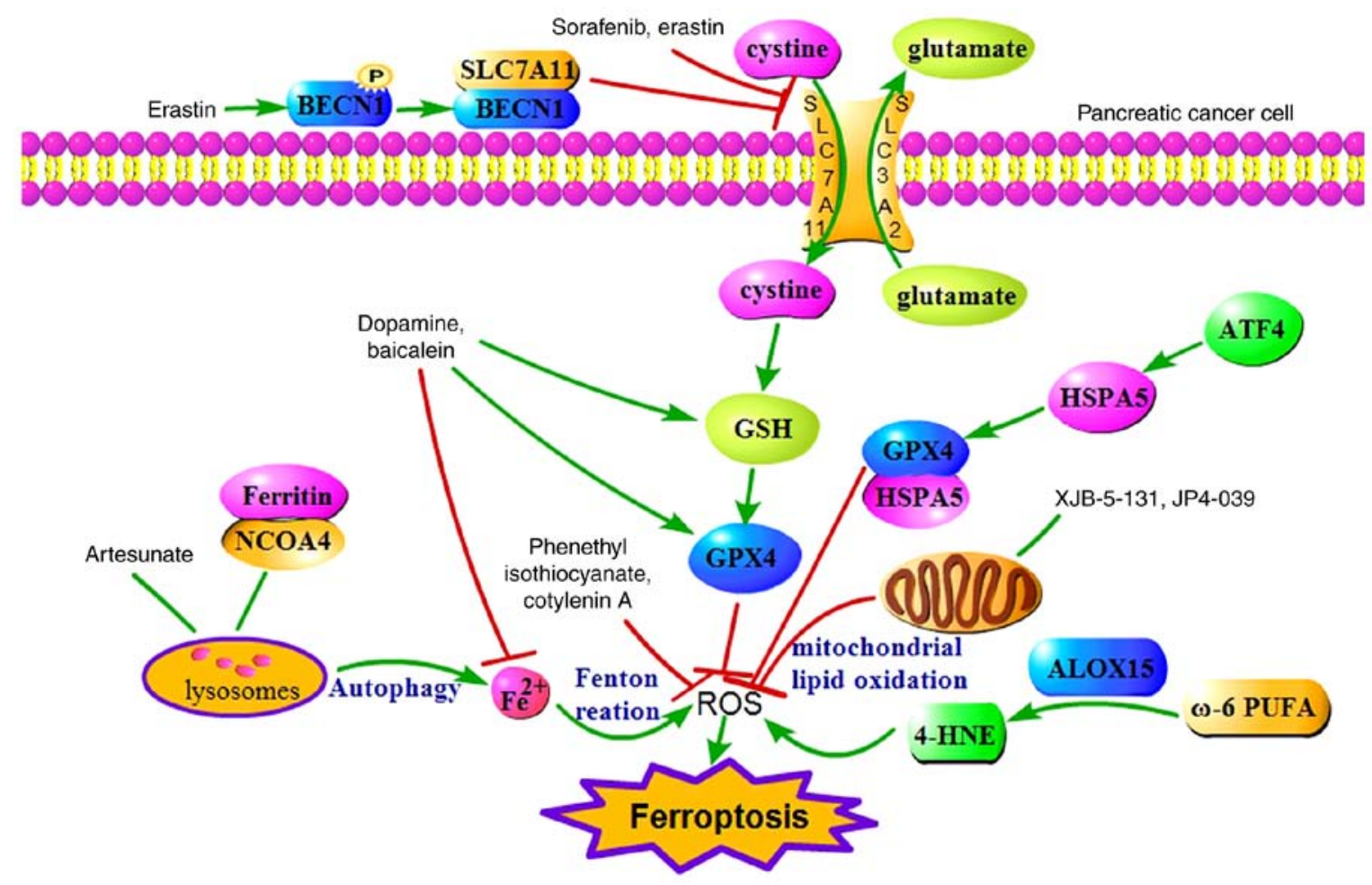

Figure 1. Potential molecular mechanism underlying ferroptosis in pancreatic cancer. Ferritinophagy, ROS metabolism and core regulators are involved in the process of ferroptosis. System Xc- and GPX4 are significant regulators of ferroptosis. Erastin and sorafenib trigger ferroptosis by inhibiting the function of system Xc-. Artesunate induces ferroptosis via ferritinophagy. ALOX15 catalyses $\omega$-6 PUFA to produce more 4-HNE, contributing to ferroptosis. ATF4-dependent HSPA5 expression inhibits ferroptosis through lipid peroxidation. Dopamine and baicalein interfere with both iron metabolism and ROS metabolism, and inhibit ferroptosis. Mitochondrially targeted nitroxide XJB-5-131 and JP4-039 inhibit ferroptosis by suppressing mitochondrial ROS accumulation. Co-treatment with cotylenin A and phenethyl isothiocyanate inhibits ROS accumulation and suppresses ferroptosis. ROS, reactive oxygen species; GPX4, glutathione peroxidase 4; GSH, glutathione; ATF4, activating transcription factor 4; HSPA5, heat shock 70 kDa protein 5; ALOX15, arachidonate 15-lipoxygenase; 4-HNE, 4-hydroxynonenal; NCOA4, nuclear receptor coactivator 4; BECN1, beclin 1.

inhibition of RAF kinase by sorafenib (70). Dixon et al (71) demonstrated that erastin treatment and silencing of SLC7A11 have similar inhibition of glutamate release. Erastin is capable of inhibiting ferroptosis by blocking cystine-glutamate exchange, as sorafenib does. Cystine is depleted due to system Xc-inhibition, which accounts for ferroptosis (71). Likewise, the study by Song et al (72) demonstrated that BECN1 plays a unique role in promoting ferroptosis in pancreatic cancer. The phosphorylation of BECN1 at Ser90/93/96 by AMP-activated protein kinase leads to the formation of the BECN1-SLC7A11 complex, which inhibits the activity of system Xc- and results in ferroptosis. In summary, inhibition of system Xc- can trigger ferroptosis in pancreatic cancer.

Ferroptosis in other associated digestive system cancers. Aside from pancreatic cancer, ferroptosis is also associated with other types of cancer of the digestive system, including hepatocellular carcinoma $(30,36,69,70,73-78)$, and colorectal cancer $(79,80)$. For example, hepatocellular carcinoma is the most common type of liver cancer, and sorafenib is the only first-line drug to treat patients with hepatocellular carcinoma. A number of studies have demonstrated that the p62-Keap1-NRF2 pathway plays a vital role in sorafenib-induced ferroptosis in hepatocellular carcinoma $(36,81,82)$. p62 suppresses the degradation of NRF2 by inactivating Keap1 and accounts for the accumulation of NRF2. Expression of NRF2 increases the transcription of quinone oxidoreductase 1, haem oxygenase-1 and ferritin heavy chain 1, bringing about resistance to ferroptosis (36). On the one hand, the retinoblastoma protein and NRF2 inhibit sorafenib-induced ferroptosis; on the other hand, haloperidol promotes sorafenib-induced ferroptosis, which provides a new therapeutic approach for hepatocellular carcinoma. Furthermore, certain ferroptosis regulators, for example, CDGSH iron sulfur domain 1, low-density lipoprotein-docosahexaenoic acid and acyl-CoA synthetase long-chain family member 4 , also regulate ferroptosis in hepatocellular carcinoma via lipid metabolism $(30,74,77)$. Table I presents studies to compare ferroptosis in pancreatic cancer, hepatocellular carcinoma and colorectal cancer.

\section{Summary and perspective}

Recently, a new iron-dependent and ROS-dependent non-apoptotic form of cell death, called ferroptosis, has been reported. The present review discusses the significant role of ferroptosis in pancreatic cancer. NCOA4-mediated ferritinophagy promotes the degradation of ferritin in lysosomes, which provides a new approach in the treatment of pancreatic cancer. In addition to iron metabolism, ROS metabolism also plays a pivotal role in ferroptosis. ALOX15-catalysed lipid hydroperoxide generation promotes ferroptosis in pancreatic cancer. By contrast, mitochondrial-targeted nitroxide negatively regulates ferroptosis by preventing mitochondrial lipid oxidation. Non-oxidative dopamine and baicalein interfere with both iron metabolism and 
ROS metabolism and attenuate pancreatic cancer. Furthermore, HSPA5, as a negative regulator, acts on GPX4 to inhibit ferroptosis in pancreatic cancer (Fig. 1). Although important discoveries have been revealed by these studies, there are also some limitations. The mechanisms of interaction between $\mathrm{CN}-\mathrm{A}$ and PEITC have not yet been elucidated in detail. The mechanism of the iron metabolism pathway and ROS pathway remains insufficiently comprehensive and clear, and additional studies should be performed in order to improve the current understanding. For early pancreatic cancer, surgical resection is the most effective treatment. Currently, the main treatment for advanced pancreatic cancer is chemotherapy. However, traditional chemotherapy drugs have limited effects on pancreatic cancer due to the anti-apoptotic effect of pancreatic cancer. One future direction for research is in regard to the molecules from traditional Chinese herbs (e.g., CN-A, PEITC and SSZ), which exhibit effects in resistant pancreatic cancer cells and could be used as a new therapy to treat pancreatic cancer. Another direction is to use gemcitabine, the first-line drug used in patients with advanced PDAC. HSPA5 overcomes gemcitabine resistance and improves the anticancer activity of gemcitabine by inducing ferroptosis. In brief, targeting ferroptosis could provide a new strategy to treat pancreatic cancer.

\section{Acknowledgements}

Not applicable.

\section{Funding}

The review was supported by funding from the National Science Foundation Grants of China (nos. 81160307 and 81560395), the Jiangxi Science \& Technology Pillar Program and the Science Foundation for Young Scholars of Jiangxi Province (grant no. 2007GQY1167).

\section{Availability of data and materials}

Not applicable.

\section{Authors' contributions}

GC and GG wrote the manuscript. GC made the tables and diagrams. XZ and $\mathrm{HC}$ put forward the concept, critically revised the article for intellectual content, and were responsible for the organization, revision and submission of the manuscript. All authors read and approved the final manuscript.

\section{Ethics approval and consent to participate}

Not applicable.

\section{Patient consent for publication}

Not applicable.

\section{Competing interests}

The authors declare that they have no competing interests.

\section{References}

1. Raimondi S, Maisonneuve P and Lowenfels AB: Epidemiology of pancreatic cancer: An overview. Nat Rev Gastroenterol Hepatol 6: 699-708, 2009.

2. Ferlay J, Colombet M, Soerjomataram I, Dyba T, Randi G, Bettio M, Gavin A, Visser O and Bray F: Cancer incidence and mortality patterns in europe: Estimates for 40 countries and 25 major cancers in 2018. Eur J Cancer 103: 356-387, 2018.

3. Bray F, Ferlay J, Soerjomataram I, Siegel RL, Torre LA and Jemal A: Global cancer statistics 2018: GLOBOCAN estimates of incidence and mortality worldwide for 36 cancers in 185 countries. CA Cancer J Clin 68: 394-424, 2018.

4. Bosetti C, Bertuccio P, Negri E, La Vecchia C, Zeegers MP and Boffetta P: Pancreatic cancer: Overview of descriptive epidemiology. Mol Carcinog 51: 3-13, 2012.

5. Kamisawa T, Wood LD, Itoi T and Takaori K: Pancreatic cancer. Lancet 388: 73-85, 2016.

6. Kim D, Zhu H, Nassri A, Mokdad A, Kukreja S, Polanco P, Huerta S and Ramzan Z: Survival analysis of veteran patients with pancreatic cancer. J Dig Dis 17: 399-407, 2016.

7. Lakatos G, Balázs A, Kui B, Gódi S, Szücs Á, Szentesi A, Szentkereszty Z, Szmola R, Kelemen D, Papp R, et al: Pancreatic cancer: Multicenter prospective data collection and analysis by the hungarian pancreatic study group. J Gastrointestin Liver Dis 25: 219-225, 2016.

8. Burris HA III, Moore MJ, Andersen J, Green MR, Rothenberg ML, Modiano MR, Cripps MC, Portenoy RK, Storniolo AM, Tarassoff P, et al: Improvements in survival and clinical benefit with gemcitabine as first-line therapy for patients with advanced pancreas cancer: A randomized trial. J Clin Oncol 15: 2403-2413, 1997.

9. Matsuda T and Matsuda A: Five-year relative survival rate of pancreas cancer in the USA, Europe and Japan. Jpn J Clin Oncol 44: 398-399, 2014.

10. Dixon SJ, Lemberg KM, Lamprecht MR, Skouta R, Zaitsev EM, Gleason CE, Patel DN, Bauer AJ, Cantley AM, Yang WS, et al: Ferroptosis: An iron-dependent form of nonapoptotic cell death. Cell 149: 1060-1072, 2012.

11. Do Van B, Gouel F, Jonneaux A, Timmerman K, Gelé P, Pétrault M, Bastide M, Laloux C, Moreau C, Bordet R, et al: Ferroptosis, a newly characterized form of cell death in parkinson's disease that is regulated by PKC. Neurobiol Dis 94: 169-178, 2016.

12. Lu B, Chen XB, Ying MD, He QJ, Cao J and Yang B: The role of ferroptosis in cancer development and treatment response. Front Pharmacol 8: 992, 2017.

13. Xie Y, Song X, Sun X, Huang J, Zhong M, Lotze MT, Zeh HJ Rd, Kang R and Tang D: Identification of baicalein as a ferroptosis inhibitor by natural product library screening. Biochem Biophys Res Commun 473: 775-780, 2016.

14. Eling N, Reuter L, Hazin J, Hamacher-Brady A and Brady NR: Identification of artesunate as a specific activator of ferroptosis in pancreatic cancer cells. Oncoscience 2: 517-532, 2015.

15. Kasukabe T, Honma Y, Okabe-Kado J, Higuchi Y, Kato N and Kumakura S: Combined treatment with cotylenin a and phenethyl isothiocyanate induces strong antitumor activity mainly through the induction of ferroptotic cell death in human pancreatic cancer cells. Oncol Rep 36: 968-976, 2016.

16. Xie Y, Hou W, Song X, Yu Y, Huang J, Sun X, Kang R and Tang D: Ferroptosis: Process and function. Cell Death Differ 23: 369-379, 2016.

17. Latunde-Dada GO: Ferroptosis: Role of lipid peroxidation, iron and ferritinophagy. Biochim Biophys Acta Gen Subj 1861: 1893-1900, 2017.

18. Dixon SJ and Stockwell BR: The role of iron and reactive oxygen species in cell death. Nat Chem Biol 10: 9-17, 2014.

19. Torti SV and Torti FM: Cellular iron metabolism in prognosis and therapy of breast cancer. Crit Rev Oncog 18: 435-448, 2013.

20. Kazan HH, Urfali-Mamatoglu C and Gunduz U: Iron metabolism and drug resistance in cancer. Biometals 30: 629-641, 2017.

21. Torti SV and Torti FM: Iron and cancer: More ore to be mined. Nat Rev Cancer 13: 342-355, 2013.

22. Yang WS, SriRamaratnam R, Welsch ME, Shimada K, Skouta R, Viswanathan VS, Cheah JH, Clemons PA, Shamji AF, Clish CB, et al: Regulation of ferroptotic cancer cell death by GPX4. Cell 156: 317-331, 2014.

23. Cao JY and Dixon SJ: Mechanisms of ferroptosis. Cell Mol Life Sci 73: 2195-2209, 2016. 
24. Yagoda N, von Rechenberg M, Zaganjor E, Bauer AJ, Yang WS, Fridman DJ, Wolpaw AJ, Smukste I, Peltier JM, Boniface JJ, et al: RAS-RAF-MEK-dependent oxidative cell death involving voltage-dependent anion channels. Nature 447: 864-868, 2007.

25. Lemasters JJ: Evolution of voltage-dependent anion channel function: From molecular sieve to governator to actuator of ferroptosis. Front Oncol 19: 303, 2017.

26. Thomas $\mathrm{T}$ and Thomas TJ: Polyamine metabolism and cancer J Cell Mol Med 7: 113-126, 2003.

27. Hu W, Zhang C, Wu R, Sun Y,Levine A and Feng Z: Glutaminase 2 , a novel p53 target gene regulating energy metabolism and antioxidant function. Proc Natl Acad Sci USA 107: 7455-7460, 2010.

28. Kang R, Kroemer G and Tang D: The tumor suppressor protein p53 and the ferroptosis network. Free Radic Biol Med 133: 162-168, 2018.

29. Linkermann A, Skouta R, Himmerkus N, Mulay SR, Dewitz C, De Zen F, Prokai A, Zuchtriegel G, Krombach F, Welz PS, et al: Synchronized renal tubular cell death involves ferroptosis. Proc Natl Acad Sci USA 111: 16836-16841, 2014.

30. Yuan H, Li X, Zhang X, Kang R and Tang D: Identification of ACSL4 as a biomarker and contributor of ferroptosis. Biochem Biophys Res Commun 478: 1338-1343, 2016.

31. Gao M, Monian P, Quadri N, Ramasamy R and Jiang X: Glutaminolysis and transferrin regulate ferroptosis. Mol Cell 59: 298-308, 2015

32. Lőrincz T, Jemnitz K, Kardon T, Mandl J and Szarka A: Ferroptosis is involved in acetaminophen induced cell death. Pathol Oncol Res 21: 1115-1121, 2015

33. Kang Y, Tiziani S, Park G, Kaul M and Paternostro G: Cellular protection using Flt 3 and PI $3 \mathrm{~K} \alpha$ inhibitors demonstrates multiple mechanisms of oxidative glutamate toxicity. Nat Commun 5: 5 , 2014.

34. Ma S, Henson ES, Chen Y and Gibson SB: Ferroptosis is induced following siramesine and lapatinib treatment of breast cancer cells. Cell Death Dis 7: e2307, 2016.

35. Roh JL, Kim EH, Jang H and Shin D: Nrf2 inhibition reverses the resistance of cisplatin-resistant head and neck cancer cells to artesunate-induced ferroptosis. Redox Biol 11: 254-262, 2017.

36. Sun X, Ou Z, Chen R, Niu X, Chen D, Kang R and Tang D: Activation of the p62-Keap1-NRF2 pathway protects against ferroptosis in hepatocellular carcinoma cells. Hepatology 63: 173-184, 2016

37. Greenshields AL, Shepherd TG and Hoskin DW: Contribution of reactive oxygen species to ovarian cancer cell growth arrest and killing by the anti-malarial drug artesunate. Mol Carcinog 56: 75-93, 2017.

38. Zhu S, Zhang Q, Sun X, Zeh HJ III, Lotze MT, Kang R and Tang D: HSPA 5 regulates ferroptotic cell death in cancer cells. Cancer Res 77: 2064-2077, 2017.

39. Gao M, Monian P, Pan Q, Zhang W, Xiang J and Jiang X: Ferroptosis is an autophagic cell death process. Cell Res 26 1021-1032, 2016

40. Tang M, Chen Z, Wu D and Chen L: Ferritinophagy/ferroptosis: Iron-related newcomers in human diseases. J Cell Physiol 233: 9179-9190, 2018

41. Mancias JD, Wang X, Gygi SP, Harper JW and Kimmelman AC: Quantitative proteomics identifies NCOA4 as the cargo receptor mediating ferritinophagy. Nature 509: 105-109, 2014.

42. Hou W, Xie Y, Song X, Sun X, Lotze MT, Zeh HJ III, Kang R and Tang D: Autophagy promotes ferroptosis by degradation of ferritin. Autophagy 12: 1425-1428, 2016.

43. Theil EC: Iron, ferritin, and nutrition. Ann Rev Nutr 24: 327-343, 2004 .

44. Yang ND, Tan SH, Ng S, Shi Y, Zhou J, Tan KS, Wong WS and Shen HM: Artesunate induces cell death in human cancer cells via enhancing lysosomal function and lysosomal degradation of ferritin. J Biol Chem 289: 33425-33441, 2014

45. Ooko E, Saeed ME, Kadioglu O, Sarvi S, Colak M, Elmasaoudi K, Janah R, Greten HJ and Efferth T: Artemisinin derivatives induce iron-dependent cell death (ferroptosis) in tumor cells Phytomedicine 22: 1045-1054, 2015.

46. Torii S, Shintoku R, Kubota C, Yaegashi M, Torii R, Sasaki M, Suzuki T, Mori M, Yoshimoto Y, Takeuchi T and Yamada K: An essential role for functional lysosomes in ferroptosis of cancer cells. Biochem J 15: 769-777, 2016.

47. Haeggström JZ and Funk CD: Lipoxygenase and leukotriene pathways: Biochemistry, biology, and roles in disease. Chem Rev 111: 5866-5898, 2011.
48. Yin $\mathrm{H}, \mathrm{Xu} \mathrm{L}$ and Porter NA: Free radical lipid peroxidation: Mechanisms and analysis. Chem Rev 111: 5944-5972, 2011

49. Shah R, Shchepinov MS and Pratt DA: Resolving the role of lipoxygenases in the initiation and execution of ferroptosis. ACS Cent Sci 4: 387-396, 2018

50. Shintoku R, Takigawa Y, Yamada K, Kubota C, Yoshimoto Y, Takeuchi T, Koshiishi I and Torii S: Lipoxygenase-mediated generation of lipid peroxides enhances ferroptosis induced by erastin and RSL3. Cancer Sci 108: 2187-2194, 2017.

51. Kuhn H, Banthiya S and van Leyen K: Mammalian lipoxygenases and their biological relevance. Biochim Biophys Acta 1851: 308-330, 2015 .

52. Wipf P, Xiao J, Jiang J, Belikova NA, Tyurin VA, Fink MP and Kagan VE: Mitochondrial targeting of selective electron scavengers: Synthesis and biological analysis of hemigramicidin-TEMPO conjugates. J Am Chem Soc 127: 12460-12461, 2005.

53. Ji J, Kline AE, Amoscato A, Samhan-Arias AK, Sparvero LJ, Tyurin VA, Tyurina YY, Fink B, Manole MD, Puccio AM, et al: Lipidomics identifies cardiolipin oxidation as a mitochondrial target for redox therapy of brain injury. Nat Neurosci 15: 1407-1413, 2012.

54. Krainz T, Gaschler MM, Lim C, Sacher JR, Stockwell BR and Wipf P: A mitochondrial-targeted nitroxide is a potent inhibitor of ferroptosis. ACS Cent Sci 2: 653-659, 2016.

55. Yen GC and Hsieh CL: Antioxidant effects of dopamine and related compounds. Biosci Biotechnol Biochem 61: 1646-1649, 1997.

56. Tarazi FI: Neuropharmacology of dopamine receptors: Implications in neuropsychiatric diseases. J Sci Res Med Sci 3 93-104, 2001.

57. Wang D, Peng Y, Xie Y, Zhou B, Sun X, Kang R and Tang D: Antiferroptotic activity of non-oxidative dopamine. Biochem Biophys Res Commun 480: 602-607, 2016.

58. Yamaguchi Y, Kasukabe T and Kumakura S: Piperlongumine rapidly induces the death of human pancreatic cancer cells mainly through the induction of ferroptosis. Int J Oncol 52: 1011-1022, 2018

59. Trachootham D, Zhou Y, Zhang H, Demizu Y, Chen Z, Pelicano H, Chiao PJ, Achanta G, Arlinghaus RB, Liu J and Huang P: Selective killing of oncogenically transformed cells through a ROS-mediated mechanism by $\beta$-phenylethyl isothiocyanate. Cancer Cell 10: 241-252, 2006.

60. Xiao D, Powolny AA, Moura MB, Kelley EE, Bommareddy A, Kim SH, Hahm ER, Normolle D, Van Houten B and Singh SV: Phenethyl isothiocyanate inhibits oxidative phosphorylation to trigger reactive oxygen species-mediated death of human prostate cancer cells. J Biol Chem 285: 26558-26569, 2010.

61. Honma Y, Kasukabe T, Yamori T, Kato N and Sassa T: Antitumor effect of cotylenin a plus interferon- $\alpha$ : Possible therapeutic agents against ovary carcinoma. Gynecol Oncol 99: 680-688, 2005.

62. Honma Y, Ishii Y, Yamamoto-Yamaguchi Y, Sassa T and Asahi K: Cotylenin A, a differentiation-inducing agent, and IFN-alpha cooperatively induce apoptosis and have an antitumor effect on human non-small cell lung carcinoma cells in nude mice. Cancer Res 63: 3659-3666, 2003.

63. Honma Y: Cotylenin A-A plant growth regulator as a differentiation-inducing agent against myeloid leukemia. Leuk Lymphoma 43: 1169-1178, 2002.

64. Li-Weber M: New therapeutic aspects of flavones: The anticancer properties of Scutellaria and its main active constituents wogonin, baicalein and baicalin. Cancer Treat Rev 35: 57-68, 2009.

65. Kurzatkowski DM and Trombetta LD: Maneb causes pro-oxidant effects in the hippocampus of Nrf2 knockout mice. Environ Toxicol Pharmacol 36: 427-436, 2013.

66. Qin S, Deng F, Wu W, Jiang L, Yamashiro T, Yano S and Hou DX: Baicalein modulates Nrf2/Keap1 system in both keap1-dependent and keap1-independent mechanisms. Arch Biochem Biophys 559: 53-61, 2014

67. Sun X, Ou Z, Xie M, Kang R, Fan Y, Niu X, Wang H, Cao L and Tang D: HSPB1 as a novel regulator of ferroptotic cancer cell death. Oncogene 34: 5617-5625, 2015.

68. Wu C: Heat shock transcription factors: Structure and regulation. Annu Rev Cell Dev Biol 11: 441-469, 1995

69. Louandre C, Ezzoukhry Z, Godin C, Barbare JC, Mazière JC, Chauffert B and Galmiche A: Iron-dependent cell death of hepatocellular carcinoma cells exposed to sorafenib. Int J Cancer 133: 1732-1742, 2013 
70. Lachaier E, Louandre C, Godin C, Saidak Z, Baert M, Diouf M, Chauffert B and Galmiche A: Sorafenib induces ferroptosis in human cancer cell lines originating from different solid tumors. Anticancer Res 34: 6417-6422, 2014.

71. Dixon SJ, Patel DN, Welsch M, Skouta R, Lee ED, Hayano M, Thomas AG, Gleason CE, Tatonetti NP, Slusher BS and Stockwell BR: Pharmacological inhibition of cystine-glutamate exchange induces endoplasmic reticulum stress and ferroptosis. Elife 3: e02523, 2014.

72. Song X, Zhu S, Chen P, Hou W, Wen Q, Liu J, Xie Y, Liu J, Klionsky DJ, Kroemer G, et al: AMPK-mediated BECN1 phosphorylation promotes ferroptosis by directly blocking system Xc-activity. Curr Biol 28: 2388-2399, 2018.

73. Bai T, Wang S, Zhao Y, Zhu R, Wang W and Sun Y: Haloperidol, a sigma receptor 1 antagonist, promotes ferroptosis in hepatocellular carcinoma cells. Biochem Biophys Res Commun 491: 919-925, 2017.

74. Yuan H, Li X, Zhang X, Kang R and Tang D: CISD1 inhibits ferroptosis by protection against mitochondrial lipid peroxidation. Biochem Biophys Res Commun 478: 838-844, 2016.

75. Chang LC, Chiang SK, Chen SE, Yu YL, Chou RH and Chang WC: Heme oxygenase-1 mediates BAY 11-7085 induced ferroptosis. Cancer Lett 416: 124-137, 2018.

76. Louandre C, Marcq I, Bouhlal H, Lachaier E, Godin C, Saidak Z, François C, Chatelain D, Debuysscher V, Barbare JC, et al: The retinoblastoma $(\mathrm{Rb})$ protein regulates ferroptosis induced by sorafenib in human hepatocellular carcinoma cells. Cancer Lett 356: 971-977, 2015.
77. Ou W, Mulik RS, Anwar A, McDonald JG, He X and Corbin IR: Low-density lipoprotein docosahexaenoic acid nanoparticles induce ferroptotic cell death in hepatocellular carcinoma. Free Radic Biol Med 112: 597-607, 2017.

78. Jennis M, Kung CP, Basu S, Budina-Kolomets A, Leu JI, Khaku S, Scott JP, Cai KQ, Campbell MR, Porter DK, et al: An african-specific polymorphism in the TP53 gene impairs p53 tumor suppressor function in a mouse model. Genes Dev 30: 918-930, 2016

79. Guo J, Xu B, Han Q, Zhou H, Xia Y, Gong C, Dai X, Li Z and Wu G: Ferroptosis: A novel anti-tumor action for cisplatin. Cancer Res Treat 50: 445-460, 2018.

80. Xie Y, Zhu S, Song X, Sun X, Fan Y, Liu J, Zhong M, Yuan H, Zhang L, Billiar TR, et al: The tumor suppressor p53 limits ferroptosis by blocking DPP4 activity. Cell Rep 20: 1692-1704, 2017.

81. Komatsu M, Kurokawa H, Waguri S, Taguchi K, Kobayashi A, Ichimura Y, Sou YS, Ueno I, Sakamoto A, Tong KI, et al: The selective autophagy substrate p62 activates the stress responsive transcription factor Nrf2 through inactivation of keap1. Nat Cell Biol 12: 213-223, 2010.

82. Suzuki T, Motohashi H and Yamamoto M: Toward clinical application of the Keap1-Nrf2 pathway. Trends Pharmacol Sci 34: 340-346, 2013.

(i) $(5)$ This work is licensed under a Creative Commons Attribution-NonCommercial-NoDerivatives 4.0 International (CC BY-NC-ND 4.0) License. 